\title{
Density Functional Theory Modeling of Twin Boundaries in CdTe as Informed by STEM Observations
}

\author{
C. Buurma ${ }^{1}$, T. Paulauskas ${ }^{1}$, Z. Guo1, R. Klie ${ }^{1}$, M. K. Y. Chan ${ }^{2} *$ \\ ${ }^{1}$ Department of Physics, University of Illinois at Chicago, Chicago, IL, USA \\ ${ }^{2}$ Center for Nanoscale Materials, Argonne National Laboratory, Argonne, IL, USA \\ * Presenting author
}

CdTe is one of the most promising photovoltaic materials, currently second only to Si in market share. However, the practical efficiencies of CdTe photovoltaic cells are still significantly below the theoretical limit, indicating possible room for improvement. One aspect in which a fundamental understanding may lead to efficiency improvements is grain boundaries. Atomistic-level characterization, including microscopy and first principles modeling, is crucial in developing such a fundamental understanding.

Twin grain boundaries, in particular, are ubiquitous in Scanning Tunneling Electron Microscopy (STEM) images of CdTe. While it is known that they are likely to be electrically benign, not much details about their formation, mutual interactions, and effects on point defects, are known. In this talk, we will discuss first principles density functional theory (DFT) modeling to investigate in detail the thermodynamics of twin formation, of interactions among twins, and of interaction between twins and point defects.

We perform periodic DFT calculations on models of $<111>$ lamellar twins and microtwins using the plane-wave code VASP and the GGA-PBE exchange-correlation functional. Models of twins of different cell sizes (up to 240 atoms) are constructed which allows for different spatial separation between twins, i.e. different twin defect densities. By varying the cell dimension in the direction perpendicular to the twins and locating the strain corresponding to the minimum energy, we determine the strain induced due to the presence of the twins. We find that the induced strains are small, in the range of $0.5-0.8 \%$. We compare the minimum energy for each size to the total energy of a periodic supercell without twins and compute the formation energy per unit area. The formation energy of twins per unit area is very low, in the range of 4-6 $\mathrm{mJ} / \mathrm{m}^{2}$. Relative to the formation energies, there is nontrivial interaction energy between the adjacent twins, which exhibit a dependence on twin separation.

We also investigate the effects of twins on intrinsic point defects. Using two representative models of lamellar and microtwins, we calculated the effect of proximity to these twins on the formation energy of individual $\mathrm{Cd}$ and Te vacancies $\left(\mathrm{V}_{\mathrm{Cd}}\right.$ and $\left.\mathrm{V}_{\mathrm{Te}}\right)$, interstitials $\left(\mathrm{Cd}_{\mathrm{I}}\right.$ and $\left.\mathrm{Te}_{\mathrm{I}}\right)$, as well as anti-sites $\left(\mathrm{Cd}_{\mathrm{Te}}\right.$ and $\mathrm{Te}_{\mathrm{Cd}}$ ). We find that the proximity to these twins has no significant effects on the formation energies of all likely individual point defects, under varying synthesis conditions. In particular, the most dominant defects, $\mathrm{V}_{\mathrm{Cd}}$ and $\mathrm{Cd}_{\mathrm{I}}$, have very similar formation energies near a twin as in bulk. There is a tendency, however, for the presence of twins to shift the defect transition levels of some individual point defects.

Finally, we investigate the formation energies of pairs of point defects that are likely, including $\mathrm{V}_{\mathrm{Cd}}+\mathrm{Cd}_{\mathrm{I}}$ (Frenkel pair), $\mathrm{V}_{\mathrm{Cd}}+\mathrm{V}_{\mathrm{Te}}$ (Schottky pair), $\mathrm{Cd}_{\mathrm{Te}}+\mathrm{Te}_{\mathrm{Cd}}$. While the other pairs behave similarly near twins as they do in bulk, we find that for Frenkel pairs, there is a significant attractive interaction near a lamellar twin that is absent in bulk or near a microtwin. Such an attractive interaction may be responsible for offstoichiometry near these twins as observed in STEM [1]. 


\section{References:}

[1] The authors acknowledge funding from the Department of Energy SunShot Initiative - Bridging Research Interactions through collaborative Development Grants in Energy (BRIDGE) program, under contract number DOE-DEEE005956. Use of the Center for Nanoscale Materials was supported by the U. S. Department of Energy, Office of Science, Office of Basic Energy Sciences, under Contract No. DE-AC02-06CH11357.
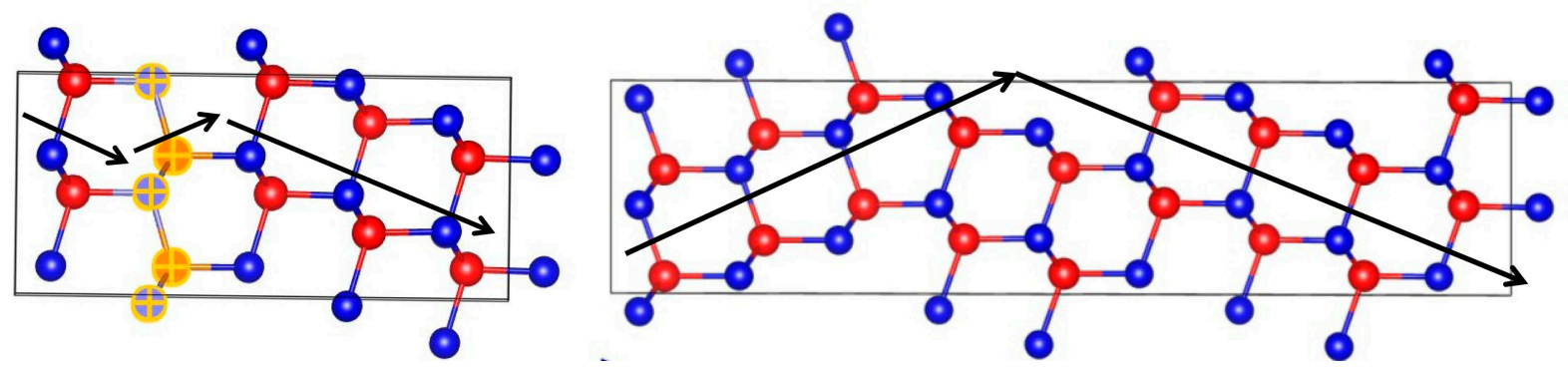

Figure 1. Two representative atomistic models of microtwin (left) and lamellar twin (right).
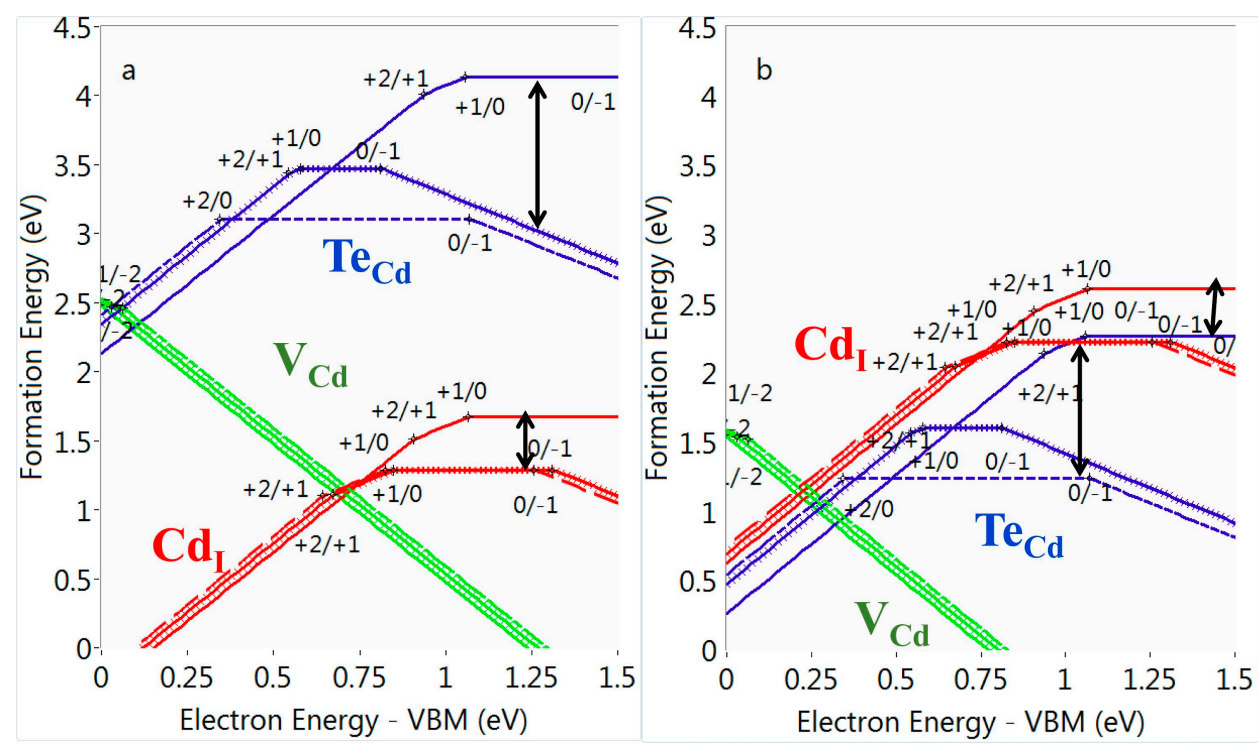

Figure 2. Formation energies of several dominant point defects in bulk (solid line) and near twins (dashed and spiked lines), under Cd-rich (left) and Te-rich (right) conditions. 